Clowes, R. C. \& Rowley, D. (1954). J. gen. Microbiol. 11, 27-33.

\title{
Genetic Investigation of the Inhibition by DL-Norleucine of the K-12 Strain of Escherichia coli
}

\author{
By R. C. CLOWES AND D. ROWLEY \\ The Wright-Fleming Institute of Microbiology, St Mary's Hospital \\ Medical School, London, W. 2
}

SUMMARY: The genetic relationship to other markers of the locus for inhibition of K-12 strain of Escherichia coli by norleucine has been investigated. The results showed little or no linkage with the loci for methionine, leucine, threonine or thiamine synthesis, but a close association was found with the Gal- and Arab- loci $_{5}^{-}$ of strain W-677.

It is probable that micro-organisms, with their presumably limited numbers of genes, will provide some of the most fruitful material available for the study of inter-action of neighbouring genes. One important aspect of this problem is whether or not the fact that two genes are adjacent on the chromosome has any biochemical significance. The mapping of the positions of various genetic loci of Escherichia coli strain K-12 is proceeding steadily, but it has not yet exceeded a purely descriptive level. One possible instance of closely linked loci having physiologically related biochemical effects has recently been reported (Manten \& Rowley, 1953); the locus for resistance to inhibition by valine in K-12 was shown to have a very high degree of linkage with that for the independence of an antagonizing amino acid, leucine.

The K-12 strain is also inhibited on minimal medium by low concentrations of norleucine, and this inhibition can be prevented by the simultaneous presence of methionine (Rowley, 1953). By marking K-12 with resistance to norleucine the genetic linkage of the locus for this character to other markers may be studied. In particular, the possibility of its close linkage with methionine independence, a tempting speculation by analogy with the linkage of valine resistance with leucine, can be investigated.

\section{MATERIALS AND METHODS}

The strains of Escherichia coli used were:

(a) $\mathrm{K}-12 / \mathbf{F}+$ (wild-type strain). This grows well in minimal medium, unsupplemented with amino acids or vitamins. Since this strain mates with F - strains, it has been presumed to be $F+$ (Hayes, 1953; Cavalli, Lederberg \& Lederberg, 1953). It is inhibited by valine $(0.1 \mu \mathrm{g} . / \mathrm{ml}$. $)$ which is reversed by either leucine $(0.05 \mu \mathrm{g} . / \mathrm{ml}$.) or isoleucine $(0.01 \mu \mathrm{g} . / \mathrm{ml}$.), and also by norleucine $(1 \mu \mathrm{g} . / \mathrm{ml}$.), which is reversed by methionine $(0 \cdot 2 \mu \mathrm{g} . / \mathrm{ml}$.$) . A stable$ mutant of this strain $\left(\mathrm{K}-12 / \mathrm{Nl}^{\mathrm{R}}\right)$ resistant to norleucine at concentrations up to $1 \mathrm{mg} . / \mathrm{ml}$., but still sensitive to valine, was selected by plating large inocula of the wild type K-12 strain on plates of minimal agar containing $1 \mathrm{mg} . / \mathrm{ml}$. 
norleucine. Both strains are capable of fermenting maltose, lactose, D-galactose, D-xylose, D-mannitol and L-arabinose $(\mathrm{Mal}+, \mathrm{Lac}+, \mathrm{Gal}+, \mathbf{X y l}+$, $\mathrm{Mtl}+, \mathrm{Arab}+$ ), shown by the production of characteristic blue-black colonies with a metallic lustre on eosin + methylene blue (EMB) agar containing any of these sugars.

(b) 58-161. A mutant of K-12 requiring methionine (20 $\mu \mathrm{g} . / \mathrm{ml}$.$) , (M-).$ $\mathbf{F}+$ and $\mathbf{F}-$ forms of this strain were used and also streptomycin-resistant mutants of both forms $\left(58-161 / \mathrm{St}^{\mathrm{R}} / \mathrm{F}-\right.$ and $\left.58-161 / \mathrm{St}^{\mathrm{R}} / \mathrm{F}+\right)$. All these strains ferment the same sugars as the parent K-12 strain.

(c) W-677. A mutant of K-12 requiring threonine $(20 \mu \mathrm{g} . / \mathrm{ml}$. $)$, leucine $(20 \mu \mathrm{g} . / \mathrm{ml}$.$) and thiamine (20 \mu \mathrm{g} . / \mathrm{ml}),.\left(\mathrm{T}^{-}, \mathrm{L}^{-}, \mathrm{B}_{\mathbf{1}}^{-}\right) . \mathrm{W}-677 / \mathrm{F}+, \mathrm{W}-677 / \mathrm{F}-$, $\mathrm{W}-677 / \mathrm{St}^{\mathrm{R}} / \mathrm{F}+$ and $\mathrm{W}-677 / \mathrm{St}^{\mathrm{R}} / \mathrm{F}$ - were used, none of which fermented the carbohydrates already mentioned, as evidenced by the production of pink colonies on EMB agar supplemented with these carbohydrates. The W-677 strains can be designated $\mathrm{Mal}^{-}, \mathrm{Lac}^{-}, \mathrm{Gal}^{-}, \mathrm{Xyl}^{-}, \mathrm{Mtl}^{-}$, $\mathrm{Arab}^{-}$.

(d) J 5-10/F + . A K-12 mutant requiring proline $(20 \mu \mathrm{g} . / \mathrm{ml}$.$) and histidine$ $\left(20 \mu \mathrm{g} . / \mathrm{ml}\right.$.) $\left(\mathrm{P}^{-}, \mathrm{H}^{-}\right)$. This strain mates with $\mathrm{F}-$ strains and is thus implied to be $\mathbf{F}+$. By plating heavy inocula on supplemented nutrient agar plates containing streptomycin $(250 \mu \mathrm{g} . / \mathrm{ml}$.) or minimal agar plates containing norleucine $\left(1 \mathrm{mg} . / \mathrm{ml}\right.$.), strains resistant to streptomycin $\left(\mathrm{J} 5-10 / \mathrm{St}^{\mathrm{B}}\right)$ and norleucine $\left(\mathrm{J} \mathrm{5-10} / \mathrm{St}^{\mathrm{R}} / \mathrm{Nl}^{\mathrm{R}}, \mathrm{J}\right.$ 5-10/Nl $\left.{ }^{\mathrm{B}}\right)$ were obtained. The $\mathrm{J5-10}$ strains are $\mathrm{Mal}+, \mathrm{Lac}+, \mathrm{Gal}+, \mathrm{Xyl}+, \mathrm{Mtl}+, \mathrm{Arab}+$.

\section{Media}

The minimal medium (MM) of Davis \& Mingioli (1950) was used throughout, supplemented with glucose or other carbohydrates. Minimal media supplemented with growth factors, e.g. $M M+B_{1}, M M+T+L$, was used in certain matings.

$E M B$ agar used in sugar fermentation tests was made up as detailed by Lederberg (1950), incorporating one of the following carbohydrates: maltose, lactose, D-galactose, D-xylose, D-mannitol or L-arabinose (supplied by Thomas Kerfoot and Co., Vale of Bardsley, Lancashire).

Nutrient agar and nutrient broth were those routinely employed in this Institute and were prepared from a tryptic digest of beef.

\section{Technique of recombination tests}

Auxotrophic technique. The two auxotrophic parent strains were grown overnight in nutrient broth at $37^{\circ}$, and, after diluting with 4 vol. of fresh broth, reincubated for $4 \mathrm{hr}$. Equal volumes of both parent cultures were then mixed, centrifuged, and the bacterial deposit resuspended in minimal medium. This procedure was repeated twice and the volume finally adjusted to $1 / 10$ that of the original; $0 \cdot 1 \mathrm{ml}$. samples were then spread over the surface of minimal or supplemented minimal agar plates in quintuplicate. Controls in duplicate of each parent were washed, concentrated, and plated separately. Colonies were isolated from the plates spread with the mixed culture after $40-48 \mathrm{hr}$. incubation at $37^{\circ}$, the 'parental' control plates being invariably devoid of colonies. 
Streptomycin prototroph technique (SRP). Overnight cultures of the parent strains, one of which was auxotrophic and streptomycin-resistant and the other prototrophic and sensitive, were diluted in fresh broth and equal volumes of the parental cultures were mixed and incubated at $37^{\circ}$ for $4 \mathrm{hr}$. After washing and concentrating as above, samples were plated on minimal medium containing streptomycin $(250 \mu \mathrm{g} . / \mathrm{ml}),.(\mathrm{MM}+\mathrm{St})$.

\section{Purification of colonies}

The surface of recombinant colonies was touched with a sterile loop and an inoculum emulsified in $\mathbf{0 . 5} \mathrm{ml}$. sterile saline to give a slight visible turbidity. A loopful of the suspension was then applied to the surface of an agar plate containing the same supplements as that from which the colony was selected. By positioning over a template, fifty colonies could be accommodated on a single agar plate which, after overnight incubation at $37^{\circ}$, was used as a source for further tests. In this way, contamination from the 'background' of the parental strains was minimized.

Sugar fermentation tests. A faintly turbid dilution in saline of each purified recombinant colony was then applied to the surfaces of EMB or MM plates containing the appropriate carbohydrate to be tested, whilst positioned over a template, so that each colony was applied on the same position on each plate.

Streptomycin and norleucine sensitivity. A loopful of the same dilution of purified recombinant colonies was streaked on freshly prepared plates of minimal agar up to ditches, containing either streptomycin $(250 \mu \mathrm{g} . / \mathrm{ml}$.$) or$ norleucine $(500 \mu \mathrm{g} . / \mathrm{ml}$.). After incubation for $24 \mathrm{hr}$., sensitive progeny were inhibited approximately $1 \mathrm{~cm}$. from the ditch, whereas resistant progeny showed growth along the whole length of the streak.

\section{RESULTS}

\section{Inhibition of parent strains}

K-12 and W-677 are inhibited by norleucine when tested on appropriately supplemented norleucine ditch plates $(500 \mu \mathrm{g} . / \mathrm{ml}$.). 58-161 shows no such inhibition. This may be due, however, to the antagonism of norleucine inhibition by the methionine which is necessary to allow growth of this auxotroph. $\mathrm{K}-12$ is also uninhibited by norleucine on this methionine-supplemented medium.

The results in Table 1 show that when the $\mathrm{F}-$ form of 58-161 is mated with a norleucine-resistant strain a proportion of the progeny are sensitive to norleucine, indicating that 58-161 carries the gene determining sensitivity to norleucine, although its action is undetectable on the methionine-containing media required for growth of this strain.

\section{Investigation of linkage to selected markers}

From the work of Hayes (1952), it is evident that in recombination there is normally a one-way transfer of genetic material. The strain which acts as a gene donor has been designated as $\mathbf{F}+$ and the strain acting as a gene 
acceptor as F - (Lederberg, Cavalli \& Lederberg, 1952). Matings can occur only when at least one of the strains is $\mathrm{F}+$. The $\mathrm{F}+$ strain, when mated with an F - strain, can be envisaged as contributing that part of its chromosome (or chromosomes) required by the $\mathbf{F}$ - parent to satisfy the requirements of the selecting medium.

When $58-161 / \mathrm{St}^{\mathrm{R}} / \mathrm{F}$ - is mated with $\mathrm{K}-12 / \mathrm{Nl}^{\mathrm{R}} / \mathrm{F}+$ using the SRP technique only $1.5 \%$ of the progeny are resistant to norleucine (Table 1, line 6). According to the above hypothesis, the $\mathrm{F}+$ parent $\left(\mathrm{K}-12 / \mathrm{Nl}^{\mathrm{R}} / \mathrm{F}+\right)$ contributes to the recombinants selected for on $\mathrm{MM}+\mathrm{St}$ that part of the chromosome which carries the factor for methionine synthesis $(\mathbf{M}+)$. Since the factor for norleucine resistance also carried by the $\mathbf{F}+$ strain is transferred to such a

Table 1. Summary of matings and sensitivity of progeny to norleucine inhibition

Mating

1. $58-161 / \mathrm{F}+\times \mathrm{W}-677 / \mathrm{F}-$

2. J5-10/F $+\times 58-161 / \mathbf{F}-$

3. $58-161 / \mathrm{F}+\times \mathrm{J} 5-10 / \mathrm{F}+$

4. $\mathrm{J} 5-10 / \mathrm{Nl}^{\mathrm{R}} / \mathrm{F}+\times 58-161 / \mathrm{F}-$

5. $\mathrm{J}_{5-10} / \mathrm{Nl}^{\mathrm{R}} / \mathrm{St}^{\mathrm{R}} / \mathrm{F}+\times \mathrm{W}-677 / \mathrm{F}-$

6. $\mathrm{K}-12 / \mathrm{Nl}^{\mathrm{R}} / \mathrm{F}+\times 58-161 / \mathrm{St}^{\mathrm{R}} / \mathrm{F}-$

7. $K-12 / N 1^{\mathrm{R}} / \mathrm{F}+\times W-677 / \mathrm{St}^{\mathrm{R}} / \mathrm{F}-$

8. $\mathrm{K}-12 / \mathrm{Nl}^{\mathrm{R}} / \mathrm{F}+\times 58-161 / \mathrm{St}^{\mathrm{R}} / \mathrm{F}+$

9. $\mathbf{K}-12 / \mathrm{Nl}^{\mathrm{R}} / \mathrm{F}+\times \mathrm{W}-677 / \mathrm{St}^{\mathrm{R}} / \mathbf{F}+$
Medium of selection

\section{MM}

MM

MM

MM

MM

$\mathbf{M M}+\mathbf{S t}$

$\mathbf{M M}+\mathbf{S t}$

$\mathbf{M M}+\mathbf{S t}+\mathrm{B}_{\mathbf{1}}$ $\mathbf{M M}+\mathbf{S t}+\mathbf{T}+\mathbf{L}$ $\mathbf{M M}+\mathbf{S t}$

$\mathbf{M M}+\mathbf{S t}$
Loci selected No. of Colonies from $\mathbf{F}+$ colonies resistant parent tested to $\mathrm{Nl}(\%)$ T, L, B 1920

M $\quad 142 \quad 0$

$\mathrm{M}$ or $\mathrm{P}, \mathrm{H} \quad 135 \quad 0$ M $100 \quad 0$ T, L, B $\quad 305 \quad 7$

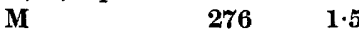
T, L, B $127 \%$ T, L $183 \quad 0$ $\begin{array}{lll}\mathrm{B}_{1} & \mathbf{8 3} & 0\end{array}$ St or M $206 \quad 13$ St or T, L, B $\quad 56 \quad 25$

small extent to these recombinants, we can deduce that there is little linkage between these two factors, or that if the $\mathrm{Nl}$ locus is linked to the $\mathrm{M}$ locus, this linkage is concealed by its closer linkage to a selected marker of the Fparent, in this case the locus for streptomycin resistance. The lack of linkage between $\mathrm{Nl}^{\mathrm{R}}$ and $\mathrm{M}+$ is corroborated by mating a Nl-resistant mutant of J 5-10/F + with 58-161/F - when of 100 progeny examined none was found to be Nl-resistant.

Similarly, the absence of significant linkage between the locus of norleucine inhibition and those for threonine, leucine or thiamine synthesis is shown by the absence of $\mathrm{Nl}^{\mathrm{R}}$ recombinants in the mating of $\mathrm{K}-12 / \mathrm{Nl}^{\mathrm{R}} / \mathrm{F}+$ and $\mathrm{W}-\mathbf{6 7 7} / \mathrm{St}^{\mathrm{R}} / \mathrm{F}$ - when the three loci for $\mathrm{T}+, \mathrm{L}+$ and $\mathrm{B}_{\mathbf{1}}+$ are selected for by mating on $\mathbf{M M}+$ St. This applies equally when these factors are separated by mating on $\mathrm{MM}+\mathrm{St}+\mathrm{B}_{1}$ (selecting for $\mathrm{T}+$ and $\mathrm{L}+$ only). The cross of $\mathrm{J} \mathrm{5}-10 / \mathrm{Nl}^{\mathrm{R}} / \mathrm{St}^{\mathrm{R}} / \mathrm{F}+\times \mathrm{W}-677 / \mathrm{F}-$ on $\mathrm{MM}$ yields only $7 \% \mathrm{Nl}^{\mathrm{R}}$ recombinants which confirms the lack of any significant linkage of $\mathrm{Nl}$ with either $\mathrm{T}, \mathrm{L}$ or $\mathbf{B}_{\mathbf{1}}$.

In these matings it is evident that the recombinants possess almost exclusively the norleucine genotype of the $\mathbf{F}$ - parent and no transfer of the norleucine locus has taken place. Matings were planned, therefore, in which the norleucine locus was selected for by incorporating the amino acid in the selecting medium. The progeny from this type of mating could then be 
examined for other unselected markers in order to compare their frequency with those from similar matings in which there was no selection for norleucine. Table 2 shows the analysis on EMB agar of the sugar fermentation markers of the progeny from the crossing of $\mathrm{K}-12 / \mathrm{Nl}^{\mathrm{R}} / \mathrm{F}+$ and $\mathrm{W}-677 / \mathrm{St}^{\mathrm{R}} / \mathrm{F}$ - when seeded on to $\mathrm{MM}+\mathrm{St}$ containing $\mathrm{Nl}(500 \mu \mathrm{g} . / \mathrm{ml}$.), which selects those recombinants in which a transfer from the $\mathbf{F}+$ to the $\mathbf{F}$ - parent of the locus for norleucine resistance $\left(\mathrm{Nl}^{\mathrm{R}}\right)$ has occurred as well as of the $\mathbf{T}+, \mathrm{L}+$ and $\mathrm{B}_{\mathbf{1}}^{+}$loci. By further supplementing the recombination plates with growth factors, recombinants could be isolated in which the norleucine locus only is selected. (The distribution of sugar fermentations of progeny from the same parents without selection of norleucine resistance is provided as comparison.)

Table 2. Effect of selection for locus for $\mathrm{Nl}$ on sugar fermentations of recombinants from the mating $\mathrm{K} 12 / \mathrm{Nl}^{\mathrm{R}} / \mathrm{F}+\times \mathrm{W}-677 / \mathrm{St}^{\mathrm{R}} / \mathrm{F}-$

\begin{tabular}{|c|c|c|c|c|c|c|c|c|}
\hline \multirow[b]{2}{*}{ Selecting medium } & \multirow{2}{*}{$\begin{array}{l}\text { No. of } \\
\text { colonies } \\
\text { tested }\end{array}$} & \multicolumn{7}{|c|}{ Percentage recombinants having the phenotype } \\
\hline & & $\mathrm{Nl}^{\mathrm{R}}$ & $\mathrm{Mal}^{+}$ & $\mathrm{Lac}^{+}$ & $\mathrm{Gal}^{+}$ & $\mathbf{X y l}^{+}$ & $\mathbf{M t l}^{+}$ & $\mathbf{A r a b}^{+}$ \\
\hline 1. $\mathbf{M M}+\mathrm{St}+\mathrm{Nl}$ & 50 & 100 & 14 & 70 & 86 & 14 & 18 & 80 \\
\hline 2. $\mathbf{M M}+\mathrm{St}+\mathrm{NI}+\mathrm{B}_{1}$ & 94 & 100 & $\mathbf{5}$ & 83 & 95 & 7 & 15 & 85 \\
\hline 3. $\mathbf{M M}+\mathbf{S t}+\mathbf{N l}+\mathbf{T}+\mathbf{L}$ & 143 & 100 & 15 & 50 & 98 & $\mathbf{2 5}$ & 28 & 82 \\
\hline 4. $\mathbf{M M}+\mathbf{S t}+\mathbf{N l}+\mathbf{T}+\mathrm{L}+\mathbf{B}_{1}$ & 184 & 100 & 15 & 38 & 93 & 27 & 26 & 73 \\
\hline 5. $\mathbf{M M}+\mathbf{S t}$ & 226 & 0 & 4 & 26 & 1 & 13 & 12 & 0 \\
\hline 6. $\mathrm{MM}+\mathrm{St}+\mathrm{B}_{1}$ & 183 & 0 & $\mathbf{1}$ & 35 & $\mathbf{0}$ & $\mathbf{3}$ & 2 & $\mathbf{3}$ \\
\hline 7. $\mathrm{MM}+\mathrm{St}+\mathrm{T}+\mathrm{L}$ & 83 & $\mathbf{0}$ & 1 & 31 & $\mathbf{0}$ & 2 & 4 & 0 \\
\hline
\end{tabular}

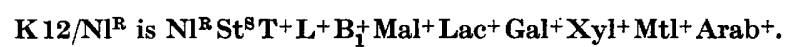

$\mathrm{W}-677 / \mathrm{St}^{\mathrm{R}} / \mathrm{F}-$ is $\mathrm{Nl}^{\mathrm{B}} \mathrm{St}^{\mathrm{R}} \mathrm{T}^{-} \mathrm{L}^{-} \mathrm{B}_{1}^{-} \mathrm{Mal}^{-} \mathrm{Lac}^{-} \mathrm{Gal}^{-} \mathrm{Xyl}^{-} \mathrm{Mtl}^{-} \mathrm{Arab}^{-}$.

The results show that selection for the locus for norleucine resistance only, as is effected when the mating is plated on minimal medium supplemented with $\mathrm{St}, \mathrm{Nl}, \mathrm{T}, \mathrm{L}$ and $\mathrm{B}_{1}$, involves an accompanying transfer of many of the sugar markers. From this it can be seen that $\mathrm{Nl}^{\mathrm{R}}$ is linked with $\mathrm{Gal}^{+}$and to a lesser extent with Arab $^{+}$.

Further evidence supporting the linkage of the locus for $\mathrm{Nl}$ inhibition with that for galactose fermentation is provided by the mating of $\mathrm{K}-12 / \mathrm{Nl}^{\mathbb{R}} / \mathrm{F}+$ and $\mathrm{W}-677 / \mathrm{St}^{\mathrm{R}} / \mathrm{F}+$ indicated in Table 1, line 9. In this mating there is no selection for Nl. Nevertheless, from fifty-six colonies tested fourteen were $\mathrm{Nl}$ resistant and each of these, and these only, was also Gal+.

\section{Further investigation of $\mathrm{Gal}^{+}$and $\mathrm{Nl}$ loci}

On selecting for the loci of $\mathrm{T}+, \mathrm{L}+$ and $\mathrm{B}_{1}^{+}$in the cross $\mathrm{K}-12 / \mathrm{Nl}^{\mathrm{R}} / \mathrm{F}+$ $\times \mathrm{W}-677 / \mathrm{S}^{\mathrm{R}} / \mathrm{F}-$, no transfer of $\mathrm{Nl}^{\mathrm{R}}$ and consequently no linkage with these factors was found. Similarly, from the inability to transfer $\mathrm{Nl}^{\mathrm{R}}$ to any significant extent in the cross $\mathrm{K}-12 / \mathrm{Nl}^{\mathrm{R}} / \mathrm{F}+\times 58-161 / \mathrm{St}^{\mathrm{R}} / \mathrm{F}-$, the inference is made of lack of linkage between $\mathrm{Nl}^{\mathrm{R}}$ and $\mathrm{M}^{+}$. From the work of Watson \& Hayes (1953), it appears that there are at least three linkage groups in the K-12 chromosome identified with the markers TL/Lac, M/B $\mathbf{B}_{1}$ and St/Mal. With the inability to localize $\mathbf{N} \mathbf{l}$ with either of the first two groups, and the fact that the Gal-locus of W-677 is also unlocalized, the possibility of the 
linkage of $\mathrm{Nl}$ and Gal factors to those of the third group, St-Mal, must be considered. For this it is necessary to call over one of the markers in the St-Mal group from the $\mathbf{F}+$ to the $\mathbf{F}-$ parent and score the progeny for the other markers.

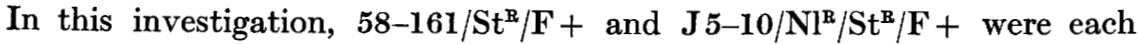
crossed with $\mathrm{W}-677 / \mathrm{F}-$. According to Hayes (1953), although $\mathrm{St}^{\mathrm{S}} \mathrm{F}+\times \mathrm{St}^{\mathrm{R}} \mathrm{F}-$ crosses are productive on $\mathbf{M M}+\mathrm{St}$, the fertility of $\mathrm{St}^{\mathrm{R}} \mathbf{F}+\times \mathrm{St}^{\mathrm{B}} \mathbf{F}-$ crosses is negligible. We found that recombinants do arise from the latter type of mating (and their number can be increased by supplementing with the F - growth factors), but the numbers of recombinants are only $2-3 \%$ of the streptomycinresistant colonies arising on minimal agar. The progeny from the cross $\mathrm{J5}-10 / \mathrm{St}^{\mathrm{R}} / \mathrm{Nl}{ }^{\mathrm{R}} / \mathrm{F}+\times \mathrm{W}-677 / \mathrm{F}$ - selected on minimal medium, and so transferring the factors $\mathrm{T}^{+}, \mathrm{L}^{+}$and $\mathrm{B}_{1}^{+}$, were scored for $\mathrm{St}^{\mathrm{R}}$ and $\mathrm{Nl}^{\mathrm{R}}$. Transfers of $29 \% \mathrm{St}^{\mathrm{R}}, 24 \% \mathrm{Mal}^{+}, 7 \% \mathrm{Nl}^{\mathrm{R}}$ and $9 \% \mathrm{Gal}^{+}$were found. Among these $\mathrm{St}^{\mathrm{R}}$ progeny, $17 \%$ were $\mathrm{Nl}^{\mathrm{R}}$ and $21 \%$ were $\mathrm{Gal}^{+}$and among the $\mathrm{St}^{\mathrm{s}}$ progeny, $4 \%$ were $\mathrm{Nl}^{\mathrm{R}}$ and $3 \% \mathrm{Gal}^{+}$. This increase in proportion of $\mathrm{Gal}^{+}$among $\mathrm{St}^{\mathrm{R}}$ progeny is substantiated in the mating $58-161 / \mathrm{St}^{\mathrm{R}} / \mathbf{F}+\times \mathbf{W}-677 / \mathbf{F}-$, where although the transfer of $\mathrm{T}^{+}, \mathrm{L}^{+}$and $\mathrm{B}_{1}^{+}$brings over only $10 \% \mathrm{St}^{\mathrm{R}}$ and $3 \% \mathrm{Gal}^{+}$, among these $\mathrm{St}^{\mathrm{R}}$ progeny there are $27 \%$ which are also $\mathrm{Gal}^{+}$. This indicates that there is a certain amount of linkage of the $\mathrm{Nl}$ and Gal loci with St.

\section{DISCUSSION}

The present work demonstrates the lack of linkage between the loci for resistance to norleucine inhibition $\left(\mathrm{Nl}^{\mathrm{R}}\right)$ and the ability to synthesize methionine $(M+)$. The previously reported linkage between resistance to valine and independence of leucine may therefore be coincidental. However, since leucine has been shown to antagonize valine only, whereas methionine is reported to antagonize inhibition by norvaline, lysine and cysteine (Rowley, 1953), the lack of linkage between $\mathrm{Nl}^{\mathrm{R}}$ and $\mathrm{M}^{+}$may be due to the non-specificity of methionine antagonism. The evidence that methionine synthesis is interrelated with that of many other amino acids and that its role in bacterial metabolism is fundamental and complex lends support to this possibility.

The linkage of the sugar markers to $\mathrm{Nl}$ is seen most clearly by comparison of results obtained by selecting for this locus with those when selection is not made (Table 2). This indicates an association of $\mathrm{Nl}$ with all the sugar markers, particularly the loci for Gal and Arab.

It will be noted from the results shown in Table 2 that the pattern of sugar markers differs from that previously published by Cavalli et al. (1953) for similar matings. These authors investigated the distribution of sugar markers from the mating of a $\mathbf{T}^{+} \mathbf{L}^{+} \mathbf{B}^{+} / \mathbf{S t}^{\mathrm{B}} / \mathbf{F}+$ strain with a $\mathbf{T}-\mathbf{L}^{-} \mathbf{B}_{\overline{\mathbf{i}}} / \mathbf{S t}^{\mathrm{R}} / \mathbf{F}-$ strain (W-945). The percentage transfer of sugar markers (scored on EMB) was found to be Arab 99.5\%, Lac 58\%, Gal 32\%, Xyl 25\% and Mal 10\%, our results from the same type of mating (Table 2, line 5) being Arab 0\%, Lac $26 \%$, Gal $1 \%$, Xyl $15 \%$ and Mal $4 \%$. The strain W-945 carries the markers $\mathrm{Mal}_{1}^{-}, \mathrm{Lac}_{1}^{-}, \mathrm{Gal}^{-}, \mathrm{Xyl}^{-}, \mathrm{Mtl}^{-}, \mathrm{Arab}^{-}$and was derived from the same parent W-1 ('T-, L-, $\left.\mathbf{B}_{\mathbf{1}}^{-}, \mathrm{Lac}_{\mathbf{1}}^{-}, \mathrm{Mal}_{\mathbf{1}}^{-}\right)$as $\mathrm{W}-677$. Thus the loci $\mathrm{Lac}_{\mathbf{1}}^{-}, \mathrm{Mal}_{\mathbf{1}}^{-}$, are allelic in the two strains, but since the markers $\mathrm{Gal}^{-}, \mathrm{Xyl}^{-}, \mathrm{Mtl}^{-}$and $\mathrm{Arab}^{-}$were 
built in separately these may or may not be allelic (E. M. Lederberg, personal communication). This possibility of non-allelism in these sugar markers led us to investigate the inability of strain W-677 to ferment these sugars. The locus $\mathrm{Gal}_{5}^{-}$carried by W-67\% allows slow growth of this strain on galactose MM (Wollman, 1953). On testing W-677 on MM incorporating either lactose, maltose, xylose, mannitol or arabinose as the sole carbohydrate source, no growth was observed except on arabinose. Strain W-945, however, was found to be unable to ferment any of the six sugars. This difference in phenotypic expression implies that the $\mathrm{Gal}^{-}$and $\mathrm{Arab}^{-}$loci may be non-allelic in these two strains. This has been confirmed by crossing $\mathrm{W}-945 / \mathrm{St}^{\mathrm{R}} / \mathrm{F}+$ with W-677 $/ \mathbf{A z}^{\mathrm{R}} / \mathrm{V}_{1}^{\mathrm{R}} / \mathbf{F}$ - on MM supplemented with $\mathrm{T}, \mathrm{L}, \mathrm{B}_{1}, \mathrm{St}, \mathrm{Az}$ and phage $\mathrm{T}_{1}$, when $5-8 \%$ of the recombinants were $\mathrm{Gal}^{+}$and $20-\mathbf{2 5} \%$ were Arab+, (L. L. Cavalli-Sforza-personal communication). The locus for fermentation of galactose carried by strain W-945 has since been characterized as $\mathrm{Gal}_{\mathbf{2}}^{-}$(E. M. Lederberg, personal communication). Hence these differences between our results (Table 2) and those of Cavalli et al. (1953) are presumably due in the case of Gal and Arab to the non-allelism of these loci in the two strains. It is the $\mathrm{Gal}_{5}^{-}$and Arab- loci of strain W-677 that are linked with Nl. Of the three linkage groups suggested by Watson \& Hayes (1953), the only one to which $\mathrm{Nl}$ shows any significant linkage is that carrying the St-Mal loci. The linkage with this group is however small, and the possibility remains that the $\mathrm{Nl}$ locus may lie in another linkage group altogether.

We are indebted to Dr W. Hayes of the Postgraduate Medical School, London, for the supply of sexually differentiated mutants, to Dr B. D. Davis, United States Public Health Service, for strain J 5-10, and to Dr L. L. Cavalli-Sforza of the Istituto Sieroterapico, Milan, for strain W-945, and permission to quote unpublished results.

\section{REFERENCES}

Cavalu, L. L., Lederberg, J. \& Lederberg, E. M. (1953). An infective factor controlling sex compatibility in Bacterium coli. J. gen. Microbiol. 8, 89.

Davis, B. D. \& Mingroli, E. S. (1950). Mutants of E. coli requiring methionine or vitamin B12. J. Bact. 60, 17.

HAYES, W. (1952). Recombinations in Bact. coli K-12: unidirectional transfer of genetic material. Nature, Lond. 169, 118.

HaYes, W. (1953). Observations on a transmissible agent determining sexual differentiation in Bacterium coli. J. gen. Microbiol. 8, 72.

LEDERBERG, J. (1950). Isolation and characterisation of biochemical mutants of bacteria. Meth. med. Res. 3, 5.

Lederberg, J., Cavaldi, L. L. \& Lederberg, E. M. (1952). Sex compatibility in Escherichia coli. Genetics, 37, 720.

Manten, A. \& Rowley, D. (1953). Genetic analysis of valine inhibition in the K12 strain of Bacterium coli. J. gen. Microbiol. 9, 226.

Rowley, D. (1953). Interrelationships between amino acids in the growth of coliform organisms. J. gen. Microbiol. 9, 37.

Watson, J. D. \& Hayes, W. (1953). Genetic exchange in Escherichia coli K-12; evidence for three linkage groups. Proc. nat. Acad. Sci., Wash. 39, 416.

Wollman, E. L. (1953). Sur le déterminisme génetique de la lysogénie. Ann. Inst. Pasteur, 84, 280.

(Received 11 December 1953) 